Théologiques

Théologiques

\title{
Mondialisation néolibérale et positions altermondialistes. Les enjeux « territoriaux " des métamorphoses du sacré
}

\section{François Houtart et Michel Beaudin}

Volume 16, numéro 1, 2008

Le territoire et le sacré

URI : https://id.erudit.org/iderudit/019186ar

DOI : https://doi.org/10.7202/019186ar

Aller au sommaire du numéro

Éditeur(s)

Faculté de théologie et de sciences des religions, Université de Montréal

ISSN

1188-7109 (imprimé)

1492-1413 (numérique)

Découvrir la revue

Citer cet article

Houtart, F. \& Beaudin, M. (2008). Mondialisation néolibérale et positions altermondialistes. Les enjeux « territoriaux » des métamorphoses du sacré. Théologiques, 16(1), 87-115. https://doi.org/10.7202/019186ar
Résumé de l'article

Cet article fait une relecture, sous l'angle des rapports entre le territoire et le sacré, des analyses plus familières de la logique et de la dynamique du capitalisme néolibéral mondialisé, comme de celles de sa réplique altermondialiste. Trois hypothèses (et leur contrepartie positive) traversent l'examen et l'interprétation de ces deux perspectives, aussi bien au plan théorique qu'à celui de leur mise en oeuvre la plus concrète : 1) une corrélation entre le caractère inégalitaire des structures d'une société et leur sacralisation (Cosmao); 2) le caractère constitutivement ternaire du rapport à l'autre (Dufour) ou du vivre ensemble sur un territoire et sa perversion sous l'égide du marché autorégulateur comme Tiers poussant à des rapports binaires de compétition et même d'exclusion; 3) une corrélation entre le durcissement du Tiers en référence sacrée - ou idole — et le sacrifice de l'autre. 


\title{
Mondialisation néolibérale et positions altermondialistes.
}

\author{
Les enjeux «territoriaux» des métamorphoses \\ du sacré
}

\author{
François HOUTART* \\ Université catholique de Louvain
}

avec la collaboration de Michel BEAUDIN, Faculté de théologie et de sciences des religions Université de Montréal

Nous nous proposons ici de reconsidérer, sous l'angle des rapports entre le sacré et la territorialité, certaines de nos analyses critiques à propos de la mondialisation néolibérale ${ }^{1}$ - phase contemporaine de l'économie capitaliste - et à propos de quelques lignes de fond émergeant du mouvement

* François Houtart est professeur émérite de sociologie à l'Université catholique de Louvain. Il est membre du Comité international du Forum social mondial, secrétaire exécutif du Forum mondial des alternatives (FMA), et président de la Ligue internationale pour les droits et la libération des peuples. Il est aussi le président-fondateur du Centre tricontinental (CETRI).

1. Le monde anglo-saxon désigne ce phénomène par le vocable de globalization. Pour certains auteurs francophones, tel le Québécois Jacques Gélinas, qui optent pour le terme "globalisation" $(2000,21)$ plutôt que pour celui de «mondialisation ", la différence serait plus que d'ordre linguistique. Ils veulent marquer que si la mondialisation des échanges économiques date déjà de plus de 500 ans, elle s'est métamorphosée récemment en une sorte de "totalitarisme économique " caractérisé par une intégration non plus seulement planétaire mais aussi "globalisante ", c'est-à-dire touchant, en plus du commerce, la production des biens et services elle-même ainsi que l'ensemble des activités humaines, et cela sous l'égide de pouvoirs économiques et d'une rationalité qui tendent à «transcender les pouvoirs étatiques » (2000, 38-51). C'est cette nouveauté que nous désignons par la qualification «néolibérale » d'une mondialisation qui déborde par ailleurs la seule sphère économique. 
altermondialiste. Dégageons d'abord quelques points de repères d'une problématique du sacré et du territoire, qui traversera notre réflexion.

\section{1. Éléments d'une problématique du sacré et du territoire}

Tout en ayant en vue une spécification par rapport au territoire, il paraît opportun de commencer par une mise en relation plus générale entre le sacré et l'organisation des rapports sociaux. Disciple du Père Louis-Joseph Lebret, o.p., et donc d'une longue de tradition d'observation et d'analyse tant des processus historiques ayant provoqué dans les pays du Sud le sousdéveloppement comme déstructuration, que des conditions favorables au développement, le théologien Vincent Cosmao énonçait, au début des années 1980, "deux lois, ou constantes, des dynamiques sociales », qui lui apparaissaient aussi comme deux "pièges » interpellant notre vigilance: «Laissées à leur inertie les sociétés se structurent dans l'inégalité. Les sociétés ne se structurent qu'en sacralisant les normes qu'elles imposent ». Et il ajoutait, en corollaire: "plus les sociétés se structurent dans l'inégalité, plus elles sacralisent leurs structures» (Cosmao 1984, 4).

Examinons cela de plus près. Cette observation pose d'abord une corrélation étroite et obligée (normes imposées) entre la structuration du vivre ensemble et les références normatives qui la rendent possible en la légitimant: constitution, lois, souverain, normes éthiques, valeurs, symboles, idéologie, références religieuses, etc. Elle souligne aussi un conditionnement mutuel entre l'aménagement concret de rapports sociaux injustes (c'est le sens que l'auteur donne ici à l'inégalité) et leur projection dans une référence ou norme qui aurait pu être "rassembleuse », mais qui, durcie, sacralisée et se prétendant exclusive (territorialisation du sacré), peut alors, en retour, aller jusqu'à conférer un caractère quasi-divin, ou "voulu par Dieu ", à un ordre établi quelconque (sacralisation du territoire).

Qu'entend-on généralement par «sacré » ? Selon l'étymologie du terme (sacer), le sacré suggère une distinction, une différence entre deux espaces, mondes ou réalités, une mise à part ou forme de «transcendance » qui peut être soit maléfique, soit bienveillante, et liée ou non à l'expérience religieuse. Le terme évoque aussi ce qui est investi d'une valeur intangible (Texier 1998, 2291-2292). L'aura du sacré fait tenir à distance et prévient toute intervention sur ce dont il se fait garant en lui prêtant son caractère propre (sacralisation «au nom de»). Par rapport à ce qui nous intéresse ici, le terme "sacré », tel que souligné chez Cosmao, implique une connotation de 
degré supplémentaire - et même de saut qualitatif et de statut absolutisé concernant toute référence ou norme sociétale.

Vincent Cosmao fait encore quelques remarques judicieuses sur notre problématique. Il observe ainsi que bien que le pouvoir comme instance de coordination ou de mise en société des humains soit "construit de mains d'hommes ", il ne s'impose à ceux-ci qu'en échappant à leur maîtrise ", qu'en revêtant un caractère sacré ou «transcendant ». «Les contraintes nécessaires au fonctionnement de la vie collective ne s'imposent, en effet, que lorsqu'elles deviennent comme évidentes, lorsqu'elles vont de soi, lorsqu'elles sont perçues comme relevant de la relation à Dieu ou aux dieux ». "Transcendance » fabriquée, donc, mais assumant un rôle d'hétéronomie marquée. Ce qui, au plan individuel, pourrait être considéré comme une perte de liberté paraît «inévitable» au plan de la dynamique sociale, comme si c'était à ce seul prix que cette référence pouvait nous représenter. "La sacralisation des organisations sociales est sans doute l'opération [...] qui contribue le plus radicalement à la structuration des sociétés ", dira encore Cosmao. En corollaire, un changement devenu nécessaire "ne peut se produire sans désacralisation des structures qui se sont figées " (Cosmao 1981, 47).

Ces perspectives rejoignent, à un plan plus formel, celles des travaux du philosophe Dany-Robert Dufour en anthropologie fondamentale. Celui-ci pose l'hypothèse d'un caractère constitutivement ternaire de la structure anthropologique du rapport à l'autre. Un rapport positif à l'autre, impliquant donc sa pleine reconnaissance, requerrait un Tiers fondateur, une référence ou une figure de l'Autre à la fois re-présentée, socialement reconnue et concrétisée, et ainsi garante du lien social qu'elle rend possible (Dufour 1990, 15-69). Grâce à la médiation de cette référence, il peut être créé du commun ou de l'inter entre l'un et l'autre et même un nous au nom duquel, par exemple, un bien commun et sa défense collective deviennent concevables. Logique ternaire, donc, comme condition et horizon de tout vivre ensemble.

Dufour fait aussi état de dérives possibles de cette structure. D’abord, celle de sa dégradation en logique unaire dans la tentative de l'un de tirer de son seul côté la référence pour s'assimiler l'autre, ou encore la dérive consistant, au nom de la même référence, à rejeter l'autre dans une pure extériorité, selon la logique binaire (l'un ou l'autre) et mortifère de l'exclusion. Il envisage même le cas d'une "altération de la fonction symbolique » ou d'une évacuation de toute référence médiatrice, laissant le rapport à l'autre dans une immédiateté néfaste, selon une logique qui caractériserait 
le néolibéralisme actuel (Dufour 2003, 15-17). On retiendra particulièrement ici que ces rapports défectueux sont avant tout une possible perversion ou détournement d'un rapport constructif, ou du «surf » fait sur le bien pour le dévoyer en mal. On remarquera aussi le rôle décisif de la manipulation et du travestissement de la référence ou du Tiers.

Faisons un pas de plus en centrant l'attention sur le rapport même à la référence. Si celle-ci se raidit, c'est-à-dire si elle cesse de s'ouvrir en renvoyant au-delà d'elle-même, à la fois vers une référence plus haute et vers l'autre, et donc en direction de sa reconnaissance et de la préservation du rapport à celui-ci, alors, elle s'absolutise et se métamorphose en idole, ce que Cosmao désignait par «sacralisation». Elle devient alors intraitable, exigeant toujours plus des humains au lieu d'être au service de leurs rapports. Il faut toujours donner plus à cette idole, qui aime se faire "prier » avant d'accorder quelque faveur. Mais l'écart, impossible à combler, se fait culpabilisant et porte à "donner" aussi l'autre à l'idole, dans l'espoir de faire le compte. La sacralisation des références entraîne donc une inversion radicale quant au rapport à l'autre. Le Tiers ne se porte plus garant de celui-ci; il ne renvoie plus l'un à l'autre, mais exige de l'un, lui-même soumis, qu'il lui sacrifie aussi l'autre (Beaudin 2006, 119-120). Comme nous le verrons, des phénomènes aussi modernes que la mondialisation n'échappent pas à une possible retombée dans la vieille ornière anthropologique et religieuse du sacrifice.

Le rapport à l'autre désigne ici, bien sûr, une formalisation du vivre ensemble sociétal que nous reprendrons, dans le cadre de cet article, sous le terme de "territoire ", en particulier dans son acception politique intégrant, sous quelque forme de souveraineté, à la fois une population, son organisation en société à tous les niveaux, et les bases matérielles ou ressources nécessaires à la vie collective. Retenons provisoirement que le «territoire » désignera inséparablement les dimensions géographique et sociale d'un processus où domine l'idée d'une délimitation de type politique (pouvoir) (Dory 1989, 2574-2575). Le territoire n'est donc pas, selon l'économiste camerounais Thierry Amougou, par exemple, un simple "espace physique neutre » ou une simple "étendue support », mais aussi

le fruit d'une nature [...], d'une histoire, d'une langue et de la traduction matérielle d'une culture. Il est la base d'un ensemble de croyances qui fondent l'identité d'un projet de vie au sein d'un système social. Il donne des habitudes particulières à ceux qui l'habitent et subit en retour leurs actions qui traduisent un rapport à l'environnement spécifique (Amougou 2008, 39). 
La considération de la territorialité est donc essentielle aux modèles comme aux modalités de développement.

Un mot, en terminant, sur les champs d'études concernés. Plusieurs recherches récentes ont mis en évidence, précisément sous l'angle du territoire, les déplacements impliqués tant par le choc de la mondialisation néolibérale, ou globalisation, que par sa réplique altermondialiste ${ }^{2}$. Du côté du sacré, plusieurs auteurs ont, depuis deux décennies au moins, abordé le capitalisme et son discours légitimateur, en particulier dans leur modalité néolibérale, sous l'angle de leur dimension quasi «religieuse », de leur caractère en quelque sorte «fondamentaliste» (Santa Ana 1991, Assmann et Hinkelammert 1993, Beaudin 1995, McCarraher 1995, Loy 1997, Folz 2007, Dufour 2007). Entendu en ce sens analogique, le «religieux» peut tout aussi bien concerner les réalités les plus séculières. Cette approche, qu'on pourrait qualifier de "religiologique ", est à distinguer de celle des analyses sociologiques sur le rôle des religions instituées et des visions spirituelles du monde, soit dans la promotion du modèle dominant de développement, soit au sein des mouvements sociaux résistant à celui-ci. On pourrait ainsi ranger sous cette dernière approche l'impact des prises de position soit de chefs d'institutions religieuses, soit de voix politiques se réclamant d'arguments confessionnels, comme celles du Président Bush l'illustrent à souhait. C'est la première perspective qui nous intéressera principalement ici en ce qui concerne le pôle du sacré.

Cependant, une approche axée spécifiquement sur les rapports entre la territorialité et le sacré, telle que proposée par ce numéro de la revue, paraît de loin plus inusitée. Nous la croyons susceptible d'une fécondité singulière. Voilà ce à quoi nous nous risquerons ici à même une analyse au plan structurel des dimensions économique, sociale, culturelle, religieuse et éthique des transformations provoquées par le coup de force néolibéral et par la résistance et les initiatives que lui oppose l'altermondialisme. Nous faisons l'hypothèse que les corrélations entre le sacré et le territoire qui animent respectivement le capitalisme néolibéral et sa contrepartie divergent radicalement et que le sort qu'elles déterminent pour les plus vulnérables, au Nord comme au Sud, constitue pour chacune le test décisif de leur humanité. En procédant ainsi, nous espérons débusquer les ressorts profonds de

2. Voir, par exemple, Territoires, développement et mondialisation, une série d'études théoriques et de cas publiée par le Centre Tricontinental (Louvain-la-Neuve) et les Éditions Syllepse (Paris) dans la revue Alternatives Sud (2008), 15/1. 
la mondialisation néolibérale et mieux enraciner l'inspiration nécessaire à son dépassement.

\section{Analyse de la mondialisation néolibérale}

La mondialisation néolibérale est bien plus que le fruit de l'évolution technologique, comme on la présente souvent comme pour en souligner le caractère inéluctable et automatiquement bienveillant, ainsi que pour la soustraire à toute velléité de remise en question ${ }^{3}$. Forcément socialement et historiquement immergée, cette orientation s'inscrit à l'intérieur d'un processus de recomposition de l'accumulation du capital, connu sous le nom de "Consensus de Washington ${ }^{4}$ » et dont les conséquences sur la dévastation de la nature et sur la destruction de la vie humaine sont, elles, aussi indéniables qu'inséparables. Désormais, un programme spécifique de développement allait s'imposer comme seule référence, à l'exclusion de tout autre, et se réservant le droit de désigner à la fois ses interprètes (FMI, OMC, etc.) et ses porteurs privilégiés (entreprises privées, surtout multinationales).

Le nouveau tournant du capitalisme se caractérise par la coordination et l'intégration mondiales des diverses étapes de la production et de la distribution en des lieux géographiques différents, en s'appuyant particulièrement sur les nouvelles techniques de la communication et de l'informatique. Cela fait de la mondialisation, comme l'écrit Michel Beaud (1999, 207), un «mouvement organique englobant». Elle a débouché sur une gigantesque concentration du pouvoir économique, de même que sur l'accroissement de la «bulle financière », facilité par l'abandon de l'étalon-or. Une telle évolution revêt des aspects économiques, sociaux, culturels et aussi proprement religieux, tout en appelant une prise de position éthique. Elle n'a pas manqué de renforcer le clivage Nord-Sud, un point de vue que nous allons privilégier ici.

3. On peut penser ici aux discours sur le capitalisme comme "fin de l'histoire " (Fukuyama), sur le marché autorégulateur (Hayek) et sur l'absence d'autre solution ( «there is no alternative», selon Madame Thatcher).

4. Il s'agit de l'accord de fait, datant de la moitié des années 1970, entre des entreprises transnationales, des organismes financiers internationaux et la Réserve fédérale des États-Unis, sur la nécessité d'orienter l'économie mondiale vers une libéralisation des marchés et une suppression des mesures régulatrices encore imposées par les États, et offrant pour cela une «boîte à outils » supposée universelle du développement. Pour un aperçu des dix principes de ce Consensus, voir Ziegler (2002, 64-65). 


\subsection{La dimension économique: mutation territoriale et nouvelle sacralité}

L'orientation néolibérale, comme stratégie de profit maximal, vise à accroître la part du capital dans les ressources produites, par rapport à celles du travail et de l'État. Ce tournant fit suite, vers le milieu des années 1970, à plus de trente ans de politique keynésienne (et fordiste) $)^{5}$ dans les sociétés occidentales, et au développement national et populiste dans la majorité des pays du Sud.

Dans les termes de notre problématique et au niveau macro-structurel, il faut comprendre ici que le capitalisme s'est d'abord développé sur des territoires locaux, élargis et intégrés plus tard à une échelle nationale. D'où la promotion, au cours de la période fordo-keynésienne, de l'État-nation comme cadre politique des activités économiques et même de l'État comme principal agent régulateur de celles-ci. Ce modèle, en favorisant une hausse constante de la demande, permit une hausse tout aussi continue des profits. Parenthèse unique dans l'histoire du capitalisme, le bien-être des populations fut couplé, au Nord, à la croissance, ce qui favorisa de nouveaux gains sociaux. Au Sud, le pacte colonial puis néocolonial s'articula aussi dans le cadre politique de l'État-nation présenté comme le promoteur central d'une «modernisation » ou du «développement », une référence «formatée » à partir des intérêts du Nord et qui impliquera une dévalorisation des pratiques et institutions économiques traditionnelles ainsi que de leur «territoire» propre (Amougou 2008), de même qu'une extraversion de ces économies.

On ne se surprendra pas qu'il en ait résulté, au Nord comme au Sud, une sacralisation du territoire "national », à travers des symboles (monnaie, hymne, drapeau, monuments, etc.), des politiques, des rites et coutumes, etc., et la fabrication d'une "identité » correspondante chez les citoyens. Cette réalité est toujours présente, comme en témoignent les frontières politiques en vigueur (terrestres et maritimes), la définition et les droits de citoyenneté, les cas de "violation» du territoire national, etc. L'ampleur du choc du 11 septembre 2001, aux États-Unis, tient en bonne partie au fait

5. Keynes était un économiste anglais qui proposa, dans les années 1930, le principe de l'intervention de l'État pour sortir des crises économiques et une plus grande participation des travailleurs. Quant à Ford, il comprit que les travailleurs, dans une économie non plus axée sur la seule offre mais aussi sur la demande, étaient aussi des consommateurs et que pour que ses ouvriers puissent acheter des voitures, il fallait accorder un salaire convenable. 
que leur territoire continental ait été touché pour la première fois, une expérience neuve dans ce pays qui avait pourtant lui-même mené des centaines d'interventions militaires à l'étranger, en Amérique latine surtout.

Mais face à une nouvelle crise de rentabilité au Nord, à partir de la fin des années 1960, et pour contrer tant les droits sociaux et la part que les populations avaient fini par acquérir sur les profits, que le mécontentement au Sud en raison des promesses trompées du modèle de «développement dépendant », les grands acteurs capitalistes prirent eux-mêmes l'initiative de remettre en question la sacralité territoriale de leurs opérations et de passer à la contre-offensive vis-à-vis du travail et de l'État. Diverses manœuvres et expériences, comme celle de la délocalisation de la production, finirent par se cristalliser dans ce qui prendra le nom de néolibéralisme, grâce au soutien d'économistes comme Hayek et Friedman — attendant depuis les années 1930 l'heure de la revanche sur Keynes -, ainsi que de certains gouvernements (Thatcher et Reagan).

L'avidité de profits toujours croissants allait maintenant passer par une libéralisation locale et par l'abolition des frontières économiques, tant pour les investissements que pour une production et des débouchés planétaires sans contraintes, et donc par une libération du capital du carcan ou des limites imposées par la société. Les accords subséquents de libre-échange, y compris en Europe, s'inscrivent dans cette dynamique d'élargissement des «pâturages» du capital.

Au Sud, la nouvelle stratégie passera d'abord par l'établissement de régimes «autoritaires », par le «cheval de Troie» de l'endettement qui permettra l'imposition de programmes d'ajustement structurel et donc une mainmise économique extérieure, laquelle rendra obsolètes les dictatures et sans risque le retour d'un État de droit ayant l'avantage de présenter une image plus légitime tout en étant privé de tout pouvoir réel. Car, au Nord comme au Sud, l'État sera déchu, au nom de la «bonne gouvernance », de ses rôles de régulateur de l'économie, de gardien du bien commun et de redistributeur de la richesse, pour être confiné à celui de l'encadrement juridique du nouvel ordre de libre marché et de stabilisateur d'une société «stressée » et souvent désolidarisée par le choc néolibéral.

Une nouvelle référence sacrée et exclusive s'impose: le marché autorégulateur, nouveau « dieu » défendant et légitimant l'appropriation privée et illimitée du nouveau territoire de "chasse » du capitalisme — aussi bien la planète entière (économie, environnement) que tout le territoire humain: génome, biens essentiels ou «insubstituables » (R. Petrella) tels l'eau, la 
terre ${ }^{6}$, le patrimoine génétique végétal, etc.; les services sociaux tels la santé et l'éducation, la sécurité sociale, les activités culturelles, etc., tous susceptibles de devenir ressources ou marchandise (Houtart 2004, 6). Le néolibéralisme tient dans ce caractère sans limite de son "territoire " et dans l'absolutisation de sa référence qui la transforme en idole destructrice.

À première vue, l'intégrité anthropologique, telle que définie ci-haut par Dufour, semble sauvegardée. Il y a bien ternarité puisque le Tiers est maintenu. Toutefois, la teneur de cette référence, le Marché, est bien celle d'une opposition radicale entre les sociétaires. Se référer à ce tiers, c'est entrer en compétition avec l'autre, jusqu'à son élimination. Il s'avère donc que le néolibéralisme n'est rien d'autre qu'une logique binaire déguisée en logique ternaire.

Voyons maintenant comment s'est déployée la nouvelle dynamique de la mondialisation sous le règne totalitaire d'une compétition ne promettant qu'aux plus forts quelque survie. Certes, la bifurcation néolibérale a permis de maintenir une croissance économique importante, mais néanmoins fragile, comme en témoignent les diverses crises. Elle encouragea un développement technologique considérable. Mais elle déboucha sur le renforcement du pouvoir d'une minorité dans le monde, avec un faible effet d'entraînement sur les couches sociales intermédiaires et le rejet de millions d'êtres humains dans la pauvreté ou l'extrême pauvreté, surtout dans le Sud. Il ne s'agit donc pas de n'importe quelle mondialisation.

Le système économique capitaliste actuel se caractérise par sa rigidité foncière, aussi bien sur le plan idéologique qu'opérationnel. Ses tenants répètent de façon si péremptoire qu'il est sans alternative viable, qu'il faut pousser la libéralisation plus avant afin de résoudre les problèmes en suspens et que la société ne saurait trouver de meilleur régulateur que le marché, qu'il serait parfois injurieux pour les religions de comparer ce discours

6. Pour prendre un seul exemple : la logique de croissance économique à tout prix bouleverse très concrètement le "territoire" des populations. Des millions de gens sont déplacés par la force de leur terre d'origine pour faire place aux exploitations pétrolières ou minières ou pour développer la monoculture industrielle ou la production d'agrocarburants. L'urbanisation sauvage est le fruit d'une telle logique. En Amérique latine, on ne s'y trompe pas: les nouveaux bidonvilles sont souvent surnommés "Friedmantown », du nom du principal économiste américain promoteur du néolibéralisme! Les populations indigènes des trois continents du Sud sont aussi chassées de leurs territoires ancestraux, pourtant inséparables de leur identité, et donc culturellement agressées. 
à leurs «dogmes ». Si du lest est jeté sur des aménagements périphériques, le fond ne reste en rien négociable.

Ainsi, les plus ouverts parmi ses partisans, tels Georges Soros ${ }^{7}$, diront qu'il faut veiller à rétablir les lois de la concurrence pour combattre les monopoles. D'autres ajouteront même que d'importants secteurs de l'activité humaine appartenant au non-marchand, comme la santé et l'éducation, doivent être protégés (Robert Reich ${ }^{8}$ ) et qu'un minimum d'État est indispensable (Michel Camdessus ${ }^{9}$ ) pour fonder efficacement le cadre légal du marché, assurer les tâches éducatives et sanitaires, réaliser les infrastructures collectives, minimiser les impacts des transformations en cours et garantir l'ordre public. Enfin, face à l'inquiétant taux de misère ou à une crise alimentaire de l'ampleur de celle qui sévit actuellement, tous les centres du pouvoir capitaliste, depuis la Banque mondiale jusqu'au Forum économique mondial de Davos, sont d'accord pour mettre sur pied des programmes de lutte contre la pauvreté, adopter des mesures d'exception et mobiliser les organisations volontaires, notamment religieuses, pour y remédier. Une fois de plus, il s'agit d'une politique assistantialiste, le pendant, ici, des initiatives privées de philanthropie, approche sans avenir et avec une tendance à incriminer les pauvres ou les perdants, surtout s'ils s'organisent pour changer les situations.

Mais n'est pas reconnu dans ces milieux le fait que le marché demeure fondamentalement un rapport social, lequel, dans le cadre du système économique existant, se fait plus structurant que jamais : il construit les inégalités et les requiert pour pouvoir se reproduire. Cela appartient à sa logique propre $^{10}$ : la concurrence, la maximisation des profits, la privatisation, la réduction incessante des coûts de production, la flexibilisation du travail et... le mérite des gagnants. Dans une course folle aux profits sans autre point de repère que la position relative des compétiteurs, de grandes entreprises multinationales n'hésitent pas à exiger des conditions de travail inhumaines dans le Tiers Monde, en imposant une désespérante spirale vers le

7. Grand spéculateur financier américain.

8. Ancien secrétaire d'État du Travail sous l'administration Clinton, démissionnaire pour raison d'éthique sociale.

9. Ancien directeur général du FMI.

10. S'adressant au Forum de Davos, en 2001, le milliardaire américain George Soros laissa échapper: "Je ne crois pas trop à l'éthique des entreprises. On ne peut leur demander de se dénaturer (We cannot ask them to jump out of their skin) ", Le Monde, 3 février 2001, cité dans J. Gélinas (2008, 172). 
bas des coûts, qui aboutit à une surexploitation des travailleurs, surtout les femmes et les enfants. Dans une telle perspective, le rapport social entre partenaires tend nécessairement à l'inégalité, principalement le rapport capital/travail ${ }^{11}$. Et le problème ne pourra que s'amplifier si le rapport mercantile continue de s'étendre normativement à l'ensemble des activités de la vie collective. La sacralisation du Tiers comme du « jeu » qu'il commande rend structurellement nécessaire le sacrifice de l'autre.

\subsection{La dimension sociale: de la promesse an sacrifice}

L'un des plus fréquents arguments, d'ailleurs très ancien, invoqué à l'appui des prétentions actuelles du capitalisme néolibéral à représenter le meilleur système possible tient, d'une part, à l'affirmation qu'il faut bien produire la richesse avant de la distribuer, ce en quoi son efficacité serait sans égale, et, d'autre part, dans la théorie du trickle down ou des retombées automatiques, à terme, pour la collectivité, des bienfaits de la richesse produite par une minorité ${ }^{12}$. On en a fait une «religion». Une telle position évite de poser deux questions fondamentales: comment se produit la richesse et comment est-elle répartie?

\subsubsection{Comment se produit la richesse}

Nul doute que la logique du capitalisme permette de produire beaucoup de richesse. Mais avec quelle contrepartie ? Au cours des 30 dernières années, près des deux tiers des forêts ont été détruites en Amérique centrale et dans une grande partie de l'Asie du Sud-Est. Le $\mathrm{CO}_{2}$ s'est accumulé dans l'atmosphère à un rythme accéléré depuis les années 70 et le réchauffement climatique s'est accéléré. Mais, on a produit de la richesse. Probablement trop au regard des besoins et des capacités environnementales, et on continue de mesurer la «santé » de l'économie en termes de taux de croissance, selon le même mode destructeur du territoire.

En 2004, les transferts du Sud vers les économies du Nord, dont elles forment la périphérie, ont dépassé 300 milliards de dollars, pour le seul

11. L'ambassadeur de l'Allemagne à Rome, M. Konrad Seitz, ne cachait pas, il y a quelques années, le "prix » d'une «restauration de la compétitivité » : " une réduction massive de la force de travail, une augmentation massive du chômage " (cité par Clairmont 1994, 18).

12. Pour un exemple de reprise contemporaine de ces théories, voir Hayek (1960, 42-45). 
service de la dette. Il s'agit donc d'un mécanisme d'absorption du «surplus économique " du Sud mis au service de la production de richesses au Nord. L'Université nationale du Mexique a calculé qu'en 22 ans, le solde des transferts du Sud vers le Nord approchait des 5 trillons de dollars (SaxeRodriguez et Nunez Rodriguez 2002a, 23), dont 2.3 trillions pour les seules Amérique latine et Caraïbes, soit plus de 10000 dollars par habitant (2002b, 233), une spoliation qui a mis fin à tout espoir de développement autocentré. Et que dire de la manipulation du «sacré » par la suspension des lois du marché lorsque leur application s'avère désavantageuse dans la concurrence avec les producteurs du Sud? Les inéquitables subventions agricoles, au Nord, les scandaleuses mesures protectionnistes des apôtres du libre-échange vis-à-vis des exportations du Sud (World Bank Institute 2008), ou encore le "dumping " vers le Sud de produits vendus à un prix bien inférieur à celui du marché dans le but d'éliminer la production locale, comme le maïs au Mexique, révèlent la véritable nature de la mondialisation néolibérale: la logique de la puissance ou de la brutalité, sans véritable «grammaire politique» ou "principe d'ordre» (Laïdi 2002, A7).

Et qu'en est-il des humains? Selon les chiffres de l'Organisation internationale du travail, il y a chaque année près de deux millions d'accidents mortels de travail, tout particulièrement dans le Sud. Le rapport indique que la cause principale est l'absence d'observation des règles de sécurité, dues en grande partie aux exigences de la production et de la compétitivité. Deux millions de vies humaines sont donc le sacrifice exigé par le moloch de la production de la richesse.

Les conditions de travail dans les entreprises de sous-traitance de l'Amérique centrale, du Sud-Est et du Sud asiatique, d'un certain nombre de pays européens de l'Est ou des pays de l'Afrique, soit de la périphérie du capitalisme central, sont semblables à celles que nous avons connues au $\mathrm{XIX}^{\mathrm{e}}$ siècle. En particulier, le travail des femmes se réalise dans des conditions déplorables: heures de travail beaucoup plus nombreuses que ce qui est légalement établi, conditions de travail infra-humaines et néfastes pour la santé, opposition à toute organisation des travailleuses, salaires inférieurs et conditions de vie à l'avenant. Mais, on crée de la richesse.

En Inde, depuis l'orientation néolibérale de l'économie, le travail des enfants s'est accru. Dans les petites entreprises de sous-traitance, les exigences de compétitivité auxquelles sont confrontées les grandes entreprises, nationales ou internationales, qui les font travailler, ont rendu plus 
prohibitifs les salaires d'adultes. D'où le recours aux enfants. Mais, entretemps, beaucoup de richesse a été produite.

Les industries pharmaceutiques n'ont pas hésité a faire un procès à l'Afrique du Sud, parce que la politique de ce pays les empêchait de répondre, comme l'a dit un des responsables à $\mathrm{CNN}$, aux exigences légitimes des actionnaires ${ }^{13}$. C'est en effet l'un des domaines de l'activité humaine les plus créateurs de richesse, et il ne fallait pas y enfreindre la loi sacro-sainte du marché.

On pourrait ainsi multiplier les exemples. Partout où le capitalisme ne rencontre pas une résistance efficace, la même visée de profit illimité et la même voie de compétition sans frein font loi. Or, ne sont pas en cause seulement des acteurs anonymes et lointains d'un nouveau capitalisme sauvage, mais bien des entreprises industrielles ou commerciales et des firmes qui ont depuis longtemps pignon sur rue chez nous. Elles s'appellent Total, Shell, Repsol, Monsanto, Rio Tinto, Coca Cola, Nike, Adidas, C\&A, etc. (Ziegler 2002). Effectivement, « laissées à leur inertie, les sociétés se structurent dans l'inégalité »(Cosmao)!

\subsubsection{Comment se distribue la richesse}

On dit que la pauvreté a diminué grâce à la croissance économique. C'est vrai en chiffres relatifs. Mais il est important de se rendre compte que cela n'est pas le résultat de la logique du système capitaliste ou de la générosité de ceux qui ont créé la richesse. Il s'agit du résultat des résistances et de l'organisation des groupes populaires qui ont conquis durement des droits sociaux et un accès plus important au produit social. Qu'on se rappelle le miracle des "tigres asiatiques », dont le coût à été payé essentiellement par deux générations de travailleurs exploités et aux droits sociaux et civiques réprimés.

Il faut ajouter aussi que la majeure partie des populations du Sud, représentant les deux tiers de l'humanité, n'est pas entrée dans un rapport réel capital/travail (par le salariat) et souffre indirectement des conséquences de la domination de la logique capitaliste, par le biais de mécanismes financiers (prix des matières premières et des produits agricoles, dette extérieure, paradis fiscaux, etc.) ou juridiques (plans d'ajustement structurel, normes

13. Un consortium des plus grandes industries pharmaceutiques a voulu interdire au gouvernement sud-africain de se procurer en Inde des médicaments génériques contre le Sida, mais ils durent retirer leur plainte, suite à l'indignation mondiale. 
de l'OMC, etc.) (Mazoyer 2002). Or, ces populations ont accompli un effort énorme d'amélioration de leur propre sort dans bien des domaines: santé, éducation, logement, etc., le plus souvent par le biais d'une économie informelle, une possibilité toujours refoulée, avec éventuellement l'appui d'organisations locales ou d'ONG, mais en dehors des circuits capitalistes ou officiels de création de la richesse. Il faut ajouter que la diminution de la pauvreté, qui est réelle en terme relatif, ne l'est nullement en chiffres absolus. À aucune époque de l'histoire, il n'y a eu autant d'êtres humains vivant dans la pauvreté (voir Alternatives Sud, 6/4, 1999). L'historien I. Wallerstein est formel à ce sujet: "Pour la proportion grandissante des forces de travail mondiales qui vivent dans les zones rurales ou migrent de ces dernières à des bidonvilles urbains, la situation est bien pire que celle de leurs ancêtres d'il y a cinq cents ans » $(1985,99)$.

La distribution des fruits de la production n'est donc pas automatiquement égalitaire, au contraire. Les modalités de production de la richesse ne prédéterminent-elles pas déjà celles de sa distribution ? La logique même du système capitaliste exige les inégalités. Or, jamais l'humanité n'a disposé d'autant de moyens pour résoudre ses problèmes. Selon un rapport du PNUD (1998, 33), un prélèvement de $4 \%$ annuel (40 milliards \$) sur la richesse cumulée des 225 plus grandes fortunes du monde permettrait la réalisation et le maintien d'un accès universel aux services sociaux de base: éducation, infrastructures sanitaires et soins de santé, eau potable, nourriture adéquate, soit les exigences minimales de la dignité humaine. Mais, la volonté politique est absente (Toussaint 1999), contrairement à l'époque keynésienne où ce relèvement de la main-d'œuvre, au Nord, était vu comme indispensable à la productivité et à la pérennité des profits.

Enfin, les écarts dans la répartition du revenu mondial s'accroissent. Selon les statistiques du PNUD (1992, 39), graphiquement représentées par la fameuse coupe de champagne, les $20 \%$ les plus riches de l'humanité absorbent $82,7 \%$ des revenus mondiaux et les $20 \%$ les plus pauvres, $1,4 \%$. C'est une expression parfaite de la logique de la répartition des richesses, une fois qu'elles sont produites dans le système économique dominant. Les écarts n'ont jamais été aussi grands entre les différentes régions du monde. Avant la révolution industrielle, ils se chiffraient entre deux et trois fois. Aujourd'hui ils atteignent un indice de 60, c'est-à-dire que le revenu moyen des régions les plus riches dépasse de 60 fois celui des régions les plus pauvres. Même dans les sociétés occidentales, les écarts se sont aussi approfondis. Aux États-Unis, par exemple, alors que les plus bas 
salaires ont eu tendance à diminuer, les plus hauts ont explosé (Stiglitz 2002). Partout, « les inégalités sociales ont bel et bien crû, quelle que soit la méthode de calcul retenue " (Laïdi 2002, A7). Contrairement aux débuts de la mondialisation néolibérale où politiciens, gestionnaires et populations ont pu être séduits à partir d'arguments essentiellement idéologiques, les échecs de celle-ci sont maintenant empiriquement documentés par les économistes (Desrosiers 2004, 1 et 8).

On peut donc s'interroger sur la répartition de la richesse une fois qu'elle a été créée. Dire qu'il faut d'abord la produire avant de la répartir est une hypocrisie considérable, car on pourrait au moins répartir celle qui existe déjà. Mais, en fait, le néolibéralisme n’a pas et ne peut avoir de projet pour les perdants. La pauvreté et l'exclusion provoquées par la loi du marché trahissent l'incapacité de celle-ci à agir comme Tiers ou à favoriser "la reconnaissance mutuelle des sujets concrets, corporels et ayant des besoins personnels et sociaux »(Hinkelammert 2000, 79), en un mot, à nous mettre en société.

\subsubsection{La dimension culturelle}

Le néolibéralisme n'est pas seulement un système économique. Il implique la référence à des valeurs et il est donc porteur de culture, en même temps qu'il véhicule un modèle de société. Les éléments auxquels nous avons fait allusion plus haut, aussi bien dans la problématique que dans l'analyse esquissée jusqu'ici, permettent déjà de s'en rendre compte.

Il y a d'abord les visions classiques provenant du siècle des Lumières, qui mirent en exergue les capacités de l'être humain de prendre en main la maîtrise de son environnement, grâce à l'application des connaissances, ce qui suscita un optimisme du progrès, base de la modernité. Parallèlement, se développaient la notion de l'individu comme porteur du projet et le caractère central de son initiative. Il fallait donc lever tous les obstacles à l'action de celui-ci. La théorie libérale en économie prit le pas sur ce qui avait été au départ une idée émancipatrice. Elle donna une dimension idéologique au développement du capitalisme.

Sa version néolibérale contemporaine, destinée à accélérer l'accumulation du capital, présenta la libéralisation des échanges des biens et des services comme la panacée des solutions aux problèmes de l'humanité contemporaine. Le premier président de l'Organisation mondiale du commerce annonçait le bonheur pour le genre humain, si cet objectif était atteint dans les 25 premières années du $\mathrm{XXI}^{\mathrm{e}}$ siècle! 
En fait, au cours des deux derniers siècles, la notion de progrès entraîna une surexploitation destructrice de la nature et de ses ressources, ainsi qu'une grave dégradation de l'environnement. Elle rendit aussi aveugle face aux injustices sociales et à la spoliation des droits sociaux provoquées par le nouveau système. Les valeurs affirmées devinrent de plus en plus illusoires, si l'on prend en compte le sort actuel des 6 milliards d'êtres humains de par le monde.

Après avoir fait éclater les références anciennes, l'État-nation et sa circonscription territoriale, le capitalisme néolibéral mondialisé bouleverse jusqu'aux notions d'espace et de temps pour les refondre dans une "territorialité concurrentielle» de tous les instants (Amougou 2008). Compétitivité, consommation, productivité et rentabilité constituent dorénavant les bases de la culture et tous ceux qui n'entrent pas dans ce modèle sont méprisés et considérés comme des foules inutiles par les porteurs de la pensée dominante. L'individualisme devient vertu et envahit tous les pores de la société. De nouveaux dogmes, des principes intouchables et leur imposition à grands renforts financiers par une rhétorique globaliste forment la base de ce que l'on a appelé «la pensée unique », tentative de conquérir le territoire humain jusque dans ses derniers retranchements intellectuels et spirituels pour le faire acquiescer à sa propre sujétion.

\subsubsection{La dimension religieuse}

Apparemment, le néolibéralisme n'a plus besoin de la religion. Aucune nécessité de justifier le rapport capital/travail par un recours à la volonté de Dieu. Au contraire, la bourgeoisie industrielle dut lutter en Europe contre l'idéologie féodale sacralisant les rapports sociaux économiques et politiques. Le capital devient, dans la nouvelle perspective, le fruit du travail et de l'intelligence et il jouit automatiquement d'une préséance de statut. Et cependant, on assiste à une véritable subversion du sacré, au sens religieux du terme, à une manipulation inattendue dans la sphère des références en y ramenant à l'avant-scène ses éléments chrétiens supposés depuis longtemps obsolètes. On pourrait à bon droit s'en étonner.

Si le recours au religieux comme tel n'est plus nécessaire, il peut cependant être utile pour donner un certain lustre aux acteurs économiques ou politiques, surtout à une époque où les mythes séculiers apparus au début de la modernité battent de l'aile, défaillants soutiens d'un ordre qui a perdu son âme. Comment un président de la Banque mondiale ou un directeur du Fonds monétaire international, qui s'affirment religieux, pourraient-ils 
prôner des politiques économiques nuisibles? Par ailleurs, le «Gott mit uns » des bannières hitlériennes et sa traduction en "God bless America » comme mentra du président George W. Bush, sans parler du dollar orné de "In God we Trust », sonnent positivement aux oreilles de nombreux publics. Les néoconservateurs aux États-Unis ont un discours proche du messianisme, où le peuple élu du XXI e siècle est celui de la nation américaine, qui doit assumer cette tâche sacrée et se donner les moyens nécessaires (surtout militaires) de l'exercer. Cela débouche sur ce qu'ils appellent un «Empire bienveillant».

Quant au discours présidentiel sur l'axe du mal, il vient ajouter à cette perspective une connotation spécifiquement religieuse, qui fait le lien avec le fondamentalisme chrétien. Ce dernier, qui interprète littéralement la Bible, applique directement les textes sacrés aux réalités historiques contemporaines, le plus souvent dans une perspective manichéenne. L'après 11 septembre 2001 a bien montré que l'invocation bushienne de Dieu et du Bien, grossièrement amalgamés, dans la religion civile américaine, à l'American way of life et à la diffusion du modèle de développement capitaliste (Beaudin 2006, 124-128), vu comme "the best way [...] to ignite a new era of global growth» (The White House 2002, 17), n'avaient d'autre but que de légitimer un coup de force contre les dernières poches de résistance à ce projet impérial, opportunément assimilées au "terrorisme ". Ces recours aux références chrétiennes viennent renforcer la trame religiologique déjà détectée dans le fondamentalisme néolibéral. On peut aussi y voir l'indice d'une structure pervertie du christianisme déjà enfouie dans l'expérience occidentale. Hier comme aujourd'hui, elles ne peuvent servir de caution aux politiques qui s'en réclament qu'au prix d'un arraisonnement de l'évangile véritable avec lequel elles divergent radicalement.

Mais ce n'est pas seulement sur le plan politique que le registre religieux vient s'inscrire. L'individualisme du néolibéralisme reçoit sa consécration des théologies du succès économique, que l'on retrouve dans certains groupes religieux pentecôtistes. Reprise sur un plan populaire, l'idée de la prédestination fait de la promesse divine un espoir d'ascension matérielle dans des couches sociales défavorisées ou vulnérabilisées. Elle rejoint une interprétation des conceptions calvinistes qui prévalut pendant longtemps dans les élites économiques des États-Unis et qui n'a pas tout à fait disparu. Ici, comme précédemment, une bataille des «dieux» comme des "théologies» semble engagée et appelle un discernement. 
Il faut ajouter aussi la dimension religieuse du postmodernisme. Ce dernier, en réaction contre le structuralisme et tous les autoritarismes, nie l'existence de systèmes, identifiant ainsi le réel avec le directement visible et place l'individu au centre d'une histoire qui ne peut être qu'immédiate (Boisvert 1996). Pour certaines conceptions et mouvements religieux, même au sein du catholicisme, cela replace le religieux en plein dans la vie, sans provoquer de conflit avec la société politique ou économique. Or, quoi de plus fonctionnel pour un système aujourd'hui mondialisé et dominant qu'une philosophie sociale niant l'existence de réalités systémiques, et si, de surcroît, d'importants courants religieux viennent de fait renforcer ce type de lecture du réel, l'effet ne peut être que plus profond. Il s'agit surtout de correspondances logiques (et pas nécessairement de complots) qui, la plupart du temps, ne remettent pas en question la sincérité des acteurs.

\subsubsection{La dimension éthique}

Face à ces situations, outre un travail théologique, il ne suffit plus de développer une éthique de condamnation des seuls abus, car la correction éventuelle de ces derniers ne pourra servir qu'à la reproduction du système. Même s'il faut continuer à signaler les abus, à les condamner et à demander réparation, il faut aller plus loin.

Il ne suffit pas non plus de proposer une éthique de régulation du système économique, car cela signifie s'adresser aux effets et non pas aux causes du phénomène. Sans doute, faut-il intervenir dans l'immédiat et les luttes pour une régulation politique des pratiques économiques, écologiques et culturelles sont indispensables. Mais la visée philosophique fondamentale d'une éthique nous oblige à aller au cœur du système et à aborder sa logique, construite au départ d'intérêts de classes (Hinkelammert 2000).

Voilà pourquoi il est nécessaire de développer une éthique critique de la philosophie de base du capitalisme qui amène ce système à créer les inégalités et les distances sociales, à faire gagner seulement le plus fort, à détruire les sources mêmes de la création des richesses (la nature et le travail humain) et finalement à ne pas répondre à l'exigence de l'économie, soit celle de fournir les bases matérielles nécessaires à la vie physique et culturelle de tous les êtres humains à travers le monde. Sur ce plan, le système capitaliste est un échec et s'est même avéré le plus inefficace que l'humanité n'ait produit. Cela conduit donc à une éthique du post-capitalisme, c'està-dire impliquant la construction d'autres références normatives et de sens, 
d'un autre système d'organisation de l'économie, obéissant à une logique ternaire véritable et qui permette de réinsérer l'économie à l'intérieur de la société (Polanyi) et selon une territorialité appropriée, plutôt que de voir la société complètement désarticulée puis réorganisée en société de marché ou sous l'empire des normes de l'économie de marché (Wallerstein 1999, 114), cette référence fut-elle symboliquement vide de sens. D’où la nécessité de trouver d'autres solutions, ce à quoi l'altermondialisme s'est attelé suite à sa réflexion critique sur l'ordre actuel.

\section{Les positions altermondialistes}

L'altermondialisme mêle étroitement les résistances à l'ordre actuel, qu'il fait converger, et des propositions et initiatives nouvelles qui pointent vers « un autre monde possible». La lutte est menée à tous les niveaux, au plan des représentations comme à celui des territoires concrets. Nous avons vu comment le capitalisme avait cherché à conforter ses assises modernes par une certaine subversion du sacré. Restons d'abord sur ce terrain pour constater comment le religieux lui-même peut aussi renverser la dynamique en subvertissant la sacralisation de la logique du Tout-Marché.

\subsection{La critique de la modernité}

Dans la foulée de notre prise de distance avec le néolibéralisme, il importe de revenir sur deux aspects qui concernent la dimension religieuse. Dans toutes les traditions, en effet, non seulement les religions construites sur une base philosophique élaborée, mais aussi les systèmes religieux des peuples indigènes - on retrouve les deux principaux piliers de toute critique de la modernité à la fois produite et véhiculée par le capitalisme.

Il s'agit d'abord du rapport à la nature où l'être humain apparaît comme faisant partie de cette dernière (la création, pour la tradition judéochrétienne) et donc appelé à vivre en symbiose avec elle plutôt que dans un rapport de domination. C'est particulièrement le cas dans les religions orientales, telles que le bouddhisme ou l'hindouisme, mais aussi du vaudou, des religions africaines ou des systèmes de croyances et de représentations des peuples autochtones des Amériques. La théologie de la libération a introduit cette dimension, avec Leonardo Boff (2000). Respecter la nature signifie le contraire de l'exploiter sans vergogne.

Le second volet est celui de la solidarité humaine, opposée à l'individualisme réducteur. Une statuette du Mozambique exprime cela avec 
force ${ }^{14}$. On y voit, dans un seul morceau de bois, un grand nombre de personnages qui forment un ensemble indissociable dans une construction collective. Toutes les traditions religieuses insistent en effet sur l'amour du prochain ou sur la compassion mutuelle et la réconciliation plutôt que sur la compétition, même si elles ne conduisent pas nécessairement à une analyse sociale très élaborée. Elles constituent donc une base de la critique de la philosophie néolibérale. Remotiver l'action des croyants pour une protestation contre les effets sociaux et écologiques désastreux du système économique et pour la recherche d'alternatives est donc un aspect important de l'altermondialisme.

Par ailleurs, la modernité met davantage l'accent sur les unités que sur les diversités et, par conséquent, tend à marginaliser la dimension du territoire, lieu des différences. Sans tomber dans le postmodernisme radical, qui voit dans l'immédiat et dans l'individu la dimension principale, sinon unique du réel, et qui privilégie donc la territorialité de manière extrême, on doit cependant reconnaître une dimension réelle à cet élément. Mais par un curieux paradoxe, le néolibéralisme a récemment favorisé l'éclatement des États et la décentralisation, sous prétexte de revaloriser le rôle de la société civile. Il ne visait nullement à redonner au territoire sa fonction de base, mais cherchait plutôt à faciliter le démantèlement de l'État par le capital pour ensuite mettre les citoyens désorganisés à la merci de ce dernier.

\subsection{La recherche d'alternatives}

Rappelons d'abord que les alternatives, qui sont le fruit des acteurs sociaux, ne peuvent surgir que de la délégitimation de la situation existante, c'està-dire du capitalisme réel (Houtart 2005). Cette étape est indispensable. En d'autres termes, il faut détruire l'idée qu'il n'y a pas d'alternatives. En effet, dans la mesure où une telle conviction reste prévalente, aucune autre solution ne sera crédible et les jeux seront faits d'avance. Sans espérance, l'imagination comme source de projets reste paralysée. D'où l'importance du rôle des instances ou références de type moral, à la fois pour ceux qui veulent réguler le système économique existant et pour ceux qui veulent le remplacer, à terme, par une autre logique.

On objectera qu'un essai d'alternative a été entrepris par le «socialisme réel » et que ce fut un échec. Certes, il faut apprendre des expériences

14. Cette statuette en bois se trouve au Centre Tricontinental à Louvain-la-Neuve (Belgique). 
du passé, mais à condition d'étudier sérieusement les causes externes et internes de l'échec et de ne pas tomber dans le simplisme d'une pensée destinée à empêcher l'émergence d'alternatives et d'une transformation qui envisage aussi le long terme. Pour aborder ce problème, il faut se situer à trois niveaux: l'utopie, les objectifs à moyen terme et les mesures immédiates. Or, à ces trois échelons, nous voyons, aujourd'hui, poindre de nombreuses idées, propositions et expérimentations. Il n'est guère difficile de noter également la convergence de ces niveaux avec une préoccupation spirituelle ou de sens.

\subsubsection{Le niveau des utopies}

Rappelons d'abord que l'utopie n'est pas illusion, mais marque un écart constitutif, en humanité, entre ce qui est et ce qui pourrait être. Il s'agit d'un projet mobilisateur: quelle société voulons-nous? Ce projet ne peut être seulement une pure construction de l'esprit. Il doit s'enraciner dans le réel, sachant qu'il s'inscrit dans un espace et dans un temps qui forment un réseau de contraintes pour les acteurs sociaux qui le mettent en œuvre.

L'utopie ou, ici, le projet mondialisateur d'abord rêvé, n'est pas définie une fois pour toutes. L'ensemble des sociétés, des peuples, des civilisations doivent constamment en retracer les contours. De plus, il ne s'agit pas de rester dans de grandes généralités, mais de se demander aussi : quelle éducation voulons-nous? quels moyens de communication (mass media)? quelle entreprise? quelle santé ? etc.

L'utopie ne saurait, par ailleurs, être définie par les seuls contenus. La donnée des acteurs importe au plus haut point, en conjonction avec le pays réel des territoires. Au delà des différences de forme, nous avons constaté la relative homogénéité et continuité qui avait fait passer le modèle de développement capitaliste du territoire national au territoire mondialisé sous l'égide de l'État puis des grandes entreprises ou Marché, la société civile d'en haut qui a confisqué le projet émancipateur de la modernité. Continuité qui a favorisé la sacralisation des références du discours.

Or, l'altermondialisme a fait entrer en scène un troisième acteur d'envergure, la société civile d'en bas, qui procède d'une tout autre logique. Elle est formée d'une myriade d'organisations de divers secteurs (paysans, ouvriers, femmes, environnementalistes, autochtones, groupes de défense des droits humains, etc.), appuyées d'ONG progressistes, et qui sont en train, à partir d'une résistance commune - bien que souvent dispersée aux atteintes du néolibéralisme à leur égard, de se coordonner en réseaux 
associatifs jusqu'au niveau mondial, au rythme même d'une prise de conscience et d'une analyse de l'ampleur des défis présents. Ce nouveau sujet historique en construction définit aussi un nouveau "territoire», terme désignant ici à la fois une localisation et une articulation des milieux de vie et d'intervention, et une logique sociale particulière. La logique singulière de cette constellation est celle du réseau, de l'horizontalité, de la participation égalitaire mais plurielle, et procédant par la convergence plutôt que par la hiérarchie et la centralisation, imposées de l'extérieur ou non. La perspective est celle d'une démocratie substantielle, aux antipodes du simple électoralisme.

Par ailleurs, si ces groupes s'attachent particulièrement à une prise en compte des besoins vitaux et du lien social en revalorisant l'identité culturelle ainsi que les savoirs et les pratiques des acteurs populaires inscrits dans un contexte spécifique (Peemans 2008, $13^{15}$ ), ils ne limitent pas à ce niveau la reterritorialisation de la maîtrise des conditions structurantes du développement et du vivre ensemble. Les données mêmes de la situation les poussent à articuler leur convergence aux niveaux régional, national et mondial tout en transigeant avec les autres acteurs et institutions tels l'État et le marché, qu'il ne s'agit pas d'abolir comme tels mais de "domestiquer ", de ramener à la raison, à leur raison d'être: le bien commun. La ligne de démarcation la plus nette, c'est que ces réseaux sont mus par «la logique de reproduction simple des conditions de la vie sociale ", par opposition à une mondialisation selon une "architecture globale unique » n'obéissant qu'à la logique de l'homogénéisation des espaces et «d'une reproduction élargie du capital sans limite» (Peemans 2008, 12-13 ${ }^{16}$ ).

En tant qu'espaces offerts aux échanges, les Forums sociaux mondiaux, régionaux et nationaux, puis thématiques et jusqu'à une Journée mondiale de mobilisation et d'action en 2008, auront contribué de façon exceptionnelle, depuis 1999, à faire converger ces réseaux et à favoriser leur conscience de former un mouvement, sans toutefois les encadrer politiquement, mais laissant à leur initiative autonome (ex. Assemblée des mouvements sociaux) le soin de proposer des orientations communes.

L'utopie de nouveaux rapports à l'autre et à la nature est en marche, aiguillonnée par la construction de Tiers ou de références communes significatives, dans le respect d'une diversité vue non pas comme un frein, mais comme une richesse à partager à même la définition collective des luttes, des

15. Pagination de la version Internet.

16. Pagination de la version Internet. 
analyses et des projets pour une mondialisation qui soit enfin bénéfique à toute l'humanité.

Pour les croyants, cela signifie une démarche qui s'inscrit dans une référence et dans une motivation religieuse, et pour les chrétiens en particulier, qui sont forts nombreux dans les forums mentionnés, il s'agit d'entrer dans la perspective des valeurs du règne de Dieu, une réalité à la fois transhistorique dans une perspective de foi, et historique dans ses traductions concrètes.

\subsubsection{Les alternatives à moyen terme}

En utilisant l'expression «à moyen terme ", nous désignons des objectifs généraux, que l'on estime atteignables, mais qui, ou bien doivent être traduits dans la complexité d'un grand nombre de propositions plus concrètes, ou bien nécessitent un long combat face aux forces d'opposition. Ils se traduisent concrètement par les projets d'une autre modulation des échanges mondialisés, par des regroupements économiques régionaux, en levant les obstacles au développement des économies dépendantes, afin de mieux répondre aux besoins des populations, en diversifiant les échanges internes et afin de constituer une base plus solide de négociation dans une économie mondialisée.

Sur un plan politique, on peut citer le renforcement des organisations internationales et leur démocratisation, mais aussi le recouvrement par l'État de sa fonction de soutien démocratique et d'intégration des initiatives de la société civile. Un tel niveau d'action, qui ne change pas radicalement le système existant, doit cependant se placer dans la perspective de son remplacement, sous peine de contribuer seulement à son adaptation et à sa reproduction. L'alternative ne se construit pas dans un vacuum. Il y aura toujours, bien sûr, des ambiguïtés à surmonter, ne serait-ce qu'en raison des pressions du système existant et de sa capacité de récupération des résistances. On peut aussi noter l'ambivalence de l'altermondialisme quant au pouvoir politique. Si les initiatives elles-mêmes restent toujours entremêlées à celles d'autres acteurs, on ne saurait cependant pas confondre les logiques respectives en présence, comme indiqué plus haut.

\subsubsection{Les alternatives à court terme}

Pour que l'on puisse parler d'alternatives crédibles, il faut non seulement se fixer un but ultime et formuler des objectifs à moyen terme, mais aussi 
faire des propositions à court terme qui puissent constituer la base d'actions revendicatrices et de programmes politiques. On ne saurait donner ici un aperçu même partiel de la myriade d'interventions de court terme, présentes et à venir, de la mouvance altermondialiste aux niveaux local et régional. Voici, cependant, quelques exemples d'initiatives ou de campagnes en cours au niveau mondial: taxation des opérations financières internationales (taxe Tobin); fiscalité régionale et internationale; suppression des paradis fiscaux; annulation de la dette des pays pauvres; protection des ressources non renouvelables et des richesses biologiques; législation internationale du travail; codes de conduite des investissements internationaux pour limiter le pouvoir discrétionnaire des entreprises multinationales; protection des productions culturelles nationales ou locales; etc.

En conclusion, des alternatives existent et d'autres surgiront encore. Qu'elles soient crédibles ne fait aucun doute. En fin de compte, leur réalisation est liée à la volonté de les mettre en œuvre. En ce moment, la crédibilité ne se pose plus au niveau de la pertinence des alternatives, mais bien à celui de l'agir collectif, lequel est intimement lié à une reconstruction anthropologique selon une logique ternaire comme nous l'avons vu précédemment. Existe-t-il des forces sociales capables de porter les projets alternatifs à court et moyen terme ? Existe-t-il une volonté politique de les réaliser? Existe-t-il une spiritualité capable d'inspirer et d'orienter les engagements ?

\section{Une spiritualité nouvelle}

Le mouvement altermondialiste est porteur de spiritualité, mais pas n'importe laquelle. S'il rejette aussi bien la pseudo-religion d'un marché sacrificiel faussement sacralisé que la subversion du sacré ou du religieux dont nous avons parlé plus haut, il n'est nullement prêt à se faire récupérer par le prosélytisme, d'où qu'il vienne. Lors des Forums sociaux mondiaux, des groupes de travail ont abordé cette question. On peut en tirer une double leçon.

Tout d'abord, il s'agit d'une spiritualité engagée, qui ne se complaît pas en elle-même, mais qui s'ouvre sur l'engagement social, en particulier en faveur des groupes sociaux les plus exploités et qui, en même temps, porte un autre regard sur la nature. À l'opposé de l'idole toujours insatiable du capital dont il conteste la "divinisation », cet engagement témoigne, chez les chrétiens par exemple, d'un Dieu qui donne avec gratuité et surabondance et qui invite à entrer dans son mouvement de partage à destination universelle. Il s'agit, ensuite, dans cette foulée, d'une spiritualité ouverte et 
pluraliste, n'excluant aucune tradition humaniste ou religieuse, sans pour autant tomber dans un syncrétisme facile; d'une spiritualité opposée aux monopoles, mais accueillante aux diversités.

Le mouvement altermondialiste commence à réaliser et à thématiser la force et même la supériorité qu'il détient sur l'Empire sur le terrain culturel, éthique et spirituel, des dimensions intégrées aux pratiques communautaires depuis souvent des millénaires (Richard 1992, 3). À une époque où sont mises en jeu la vie pour tous et la sauvegarde de la nature en raison de l'inefficacité du système à ce plan, cette force d'humanité pourrait s'avérer décisive malgré la supériorité économique, politique et militaire du capital.

C'est ainsi que lors des Forums sociaux mondiaux et continentaux, des ateliers ont abordé la théologie de la libération, le dialogue entre religions, les tâches communes en faveur de la paix, les traditions spirituelles de l'islam, du bouddhisme, de l'hindouisme, du judaïsme, du christianisme. Le Forum social de Mumbai (Inde) fut une occasion privilégiée à cet effet. Répliquer à l'instrumentalisation du religieux par le néolibéralisme ou encore aux autres formes de sacralisation que celui-ci met en œuvre ne signifie pas pour autant sacraliser le combat de l'altermondialisme, mais consiste à prendre en compte la dimension spirituelle au sein même des luttes sociales. D'abord pour faire droit aux sources religieuses et humanistes libérantes qui inspirent et donnent du souffle aux acteurs de la "remondialisation " (Dierckxens 2001, 138), et ensuite pour ne plus abandonner l'exclusivité de ce terrain à la droite néolibérale.

La redécouverte de la territorialité, dans une telle perspective, ne peut évidemment pas déboucher sur un communautarisme du repli, mais bien sur une remise en valeur d'une dimension permettant aux humains d'avoir prise sur leur quotidien tout en s'ouvrant à l'universel et de construire ainsi une logique sociale et de vie selon des références qui la fassent échapper au totalitarisme asservissant du marché et à la réduction du sacré à la loi de la valeur. Afin de faire en sorte, pour parler théologiquement à la manière de Paul, qu'en Celui qui nous a libéré de toute "dette» ou sujétion à l'égard des «Autorités » et des «Pouvoirs", l'humanité de doive plus rien à personne, sinon à elle-même (Col 2, 10-15).

\section{Références}

Amougou, T. (2008), "Territorialité politique, territorialité concurrentielle et développement », Alternatives Sud, 15/1, p. 39-68. 
Assmann, H. et F.J. Hinkelammert, (1993), L'idolâtrie de marché. Critique théologique de l'économie de marché, Paris, Cerf.

BEAUD, M., et al. (GEMDEV) (1999), La mondialisation. Les mots et les choses, Paris, Karthala.

BeAudin, M. (1995), "Cette idole qui nous gouverne. Le néolibéralisme comme 'religion' et 'théologie' sacrificielles ", Studies in Religion/Sciences religieuses, 24/4, p. 395-413.

(2006), «Le Dieu du président Bush et le Dieu des théologies de la libération sauvent-ils?", dans R. MAGER, dir., Dieu agit-il dans l'histoire?, Montréal, Fides (Héritage et projet 70), p. 115-133.

Boff, L. (2000), "Ecologie-théologie: il n'y a pas de ciel sans terre ", Alternatives Sud, 7/1, p. 243-264.

BOISVERT, Y., Le monde postmoderne: analyse du discours sur la postmodernité, Paris/Montréal, L'Harmattan, 1996.

Clairmont, F.F. (1994), «La diabolique logique de la productivité », Le Monde diplomatique, 484, p. 18 (= International Herald Tribune, 10 mars 1994).

COLLECTIF (1999), Comment se construit la pauvreté?, Alternatives Sud, 6/4.

Cosmao, V. (1981), Changer le monde. Une tâche pour l'Église, Paris, Cerf (Traditions chrétiennes).

(1984), «Problématique de la théologie de la libération », Foi et développement, 117-118, p. 1-6.

Desrosiers, É. (2004), «La mondialisation n’a pas tenu ses promesses. Les économistes ont mesuré un accroissement général des inégalités dans le monde ", Le Devoir, 24 novembre, p. 1 et 8.

(2008), «Une ombre au libre-échange », Le Devoir, 18 juin 2008, p. B1 et B4.

DierckXeNs, W. (2001), «Vers une alternative citoyenne », Alternatives Sud, 8/2, p.127-140.

Dory, D. (1989), «Territoire ", dans A. JACOB, dir., Encyclopédie philosophique universelle, v. 2 Notions philosophiques - Dictionnaire, t. 2, $M-Z$, Paris, PUF, p. 2574-2575.

Dufour, D.-R. (1990), Les mystères de la trinité, Paris, Gallimard.

(2003), L'art de réduire les têtes. Sur la nouvelle servitude de l'homme libéré à l'ère du capitalisme total, Paris, Denoël. 

Denoël.

Folz, R. (2007), "The Religion of the Market: Reflections on a Decade of Discussion ", Worldview, 11, p.135-154.

Fukuyama, F. (1992), La fin de l'histoire et le dernier homme, trad. par D.-A. Canal, Paris, Flammarion.

GÉLINAS, J. (2000), La globalisation du monde. Laisser faire ou faire?, Montréal, Écosociété.

(2008), Dictionnaire critique de la globalisation. Les mots du pouvoir, le pouvoir des mots, Montréal, Écosociété.

HaYek, F.A. Von (1960), The Constitution of Liberty, Chicago, The Chicago University Press.

Hinkelammert, F.J. (2000), "La théologie de la libération dans le contexte économique et social de l'Amérique latine ", Alternatives Sud, 7/1, p. 77-100.

Houtart, F., (2004), Que penser de la mondialisation?, Namur, Fidélité. (2005), Délégitimer le capitalisme et recréer l'espérance, Bruxelles, Collophon.

LAÏDI, Z. (2002), « 11 septembre - Une mondialisation sans grammaire politique », Le Devoir, 9 septembre, p. A7.

LoY, D. (1997), "The Religion of the Market ", Journal of the American Academy of Religion, 45/2, p. 275-290.

MAzoyer, M. (2002), "Mondialisation libérale et pauvreté paysanne quelle alternative?", Alternatives Sud, 9/4, p. 5-26.

McCarraher, E. (2005), "The Enchantments of Mammon : Notes towards a Theological History of Capitalism », Modern Theology, 21/3, p. 429461.

PeEmans, J.-P. (2008), "Territoires et mondialisation : enjeux du développement", Alternatives Sud, 15/1, p. 7-35. Version Internet: http ://www.cetri.be/spip.php ? article346\&lang=fr (consulté le 04 août 2008).

PNUD (1992), Rapport mondial sur le développement humain, Paris, Economica.

(1998), Rapport mondial sur le développement humain, Paris, Economica. 
Richard, P. (1992), "La théologie de la libération. Thèmes et défis nouveaux pour la décennie 1990 », Foi et développement, p. 1-6.

SANTA ANA, J. de (1991), La practica economica como religion. Critica teologica a la economia politica, San José (Costa Rica), DEI.

Saxe-Rodriguez, J., Nunez Rodriguez, O.R. (2002a), "Annexes ", Alternatives Sud, 9/2-3, p. 20-24. (2002b), «Le transfert des excédents du Sud vers le Nord: le cas de l'Amérique latine entre 1976 et 1997 ", Alternatives Sud, 9/2-3, p. 233239.

Stiglitz, J. (2002), La grande désillusion, Paris, Plon.

TEXIER, R. (1989), "Sacré ", dans A. JACOB, dir., Encyclopédie philosophique universelle, v. 2 Notions philosophiques - Dictionnaire, t. 2, M-Z, Paris, PUF, p. 2291-2293.

THE WhITE House (septembre 2002), The National Security Strategy of the United States of America. http ://www.whitehouse.gov/nsc.pdf (consulté le 11 juin 2004).

Toussaint, E. (1999), La Bourse ou la Vie. La finance contre les peuples, Paris, Syllepse.

Wallerstein, I. (1985), Le capitalisme historique, Paris, La Découverte (Repères 29).

(1999), L'après libéralisme. Essai sur un système-monde à réinventer, La Tour-d'Aigues, L'Aube.

World BANK InstituTe (2008), World Trade Indicators 2008 : Benchmarking and Performance, Washington (D.C.), Pap/Cdr.

ZIEGLER, J. (2002), Les nouveaux maîtres du monde et ceux qui leur résistent, Paris, Fayard. 


\section{Résumé}

Cet article fait une relecture, sous l'angle des rapports entre le territoire et le sacré, des analyses plus familières de la logique et de la dynamique du capitalisme néolibéral mondialisé, comme de celles de sa réplique altermondialiste. Trois hypothèses (et leur contrepartie positive) traversent l'examen et l'interprétation de ces deux perspectives, aussi bien au plan théorique qu'à celui de leur mise en œuvre la plus concrète : 1) une corrélation entre le caractère inégalitaire des structures d'une société et leur sacralisation (Cosmao); 2) le caractère constitutivement ternaire du rapport à l'autre (Dufour) ou du vivre ensemble sur un territoire et sa perversion sous l'égide du marché autorégulateur comme Tiers poussant à des rapports binaires de compétition et même d'exclusion; 3) une corrélation entre le durcissement du Tiers en référence sacrée —- ou idole —- et le sacrifice de l'autre.

\section{Abstract}

This paper reconsiders prevailing analyses of the logic and dynamics of globalized neoliberal capitalism and its anti-globalization counterpart from the perspective of the relationship between the notions of "land" and "sacred". Three assumptions frame the analysis and interpretation of these two alternatives, both theoretically and empirically: 1) the existence of a correlation between the degree of inequality inherent in a society's structures and their sacredness (Cosmao); 2) the constitutive role played by "land" in structuring relations to the Other (Dufour), or the ability to live together on the same land, and the perversion of this constitutive link under the aegis of a selfregulating market that transforms the tripartite relationship between man, the Other and the land into a binary relationship of competition and exclusion; 3) a correlation between the process whereby the mediating Third party becomes "sacralised" and the willingness to sacrifice the "Other". 